\title{
Formation of Satisfactory and Dissatisfactory Experiences with Augmented Reality: An Abstract
}

\author{
Atieh Poushneh and Arturo Vasquez-Parraga
}

\begin{abstract}
Augmented reality technology is intended to enhance user's experience in shopping or seeking entertainment. If enhancement is low or nonexistent, it may not be because augmented reality (AR) is faulty; it may be because AR did not provide sufficient attributes to satisfy customers. Based on a study with adult consumers, this study examines the level of satisfaction or dissatisfaction achieved by some users of AR in shopping and entertainment. The results show a significant discrepancy between what consumers expected to be offered when engaging augmented reality in shopping or entertainment and what they actually experienced. Consumers expected high levels of interactivity, high quality of augmentation (realistic view and telepresence), high levels of information, and the availability of crucial utilities (search features, narration, quick response, and need for touch). Consumers experienced fun, pleasure, and connectivity, but they also encountered low levels of interactivity and low levels of information. Important implications for researchers and managers are drawn from this discrepancy.
\end{abstract}

References Available Upon Request

A. Poushneh $(\varangle) \cdot$ A. Vasquez-Parraga University of Texas Rio Grande Valley, Edinburg, TX, USA

e-mail: atieh.poushneh01@utrgv.edu; arturo.vasquez@utrgv.edu 